\title{
Mental Imagery of Free Fall: Does a Falling Apple Accelerate in Our Minds?
}

\author{
Daniel Bratzke ${ }^{1,2, *}$ and Rolf Ulrich ${ }^{2}$ \\ 'Department of Psychology, University of Bremen, Hochschulring 18, 28359 Bremen, Germany \\ ${ }^{2}$ Department of Psychology, University of Tübingen, Schleichstraße 4, 72076 Tübingen, Germany
}

Received 21 July 2020; accepted 13 October 2020

\begin{abstract}
The present study examined whether people's mental imagery of falling objects includes the acceleration due to the earth's gravitational force. To investigate this question, we used two different tasks, a height estimation and a fall-time estimation task. In the height estimation task, participants were presented with different free-fall times and had to indicate the corresponding heights from which the object fell to the ground. In the fall-time estimation task, participants had to produce the fall time associated with free falls from different heights. In contrast to the law of free fall, our results are more consistent with a linear than with an accelerated relationship between height and fall time. Thus, the present results suggest that mental imagery of an object's free fall does not represent the gravitational acceleration due to gravity.
\end{abstract}

Keywords

Intuitive physics, mental imagery, time perception

\section{Introduction}

Over the last decades, ample evidence has been gathered demonstrating that people's intuition about the physical world is often incorrect or biased (for a review, see Kubricht et al., 2017). One example of such a misconception is the fallingobject problem: People commonly indicate that an object falling from a moving body (or airplane) should fall to the ground on a straight path rather than on a parabolic one (e.g., McCloskey et al., 1983). Other examples related to gravita-

"To whom correspondence should be addressed. E-mail: bratzke@uni-bremen.de 
tional phenomena are the mass-speed illusion (i.e., the belief that heavier objects fall faster than lighter ones; e.g., Rohrer, 2002; Vicovaro et al., 2019) and the Galileo bias (i.e., the belief that all objects fall at the same rate; Oberle et al., 2005). Another interesting aspect of free fall is gravitational acceleration. Does people's intuitive physics represent the acceleration of falling objects due to the earth's gravitational force?

For example, Shanon (1976) wondered whether people's intuitive physics of falling objects resembles the Newtonian model (i.e., they fall with constant acceleration) or the Aristotelian model (i.e., they fall with constant speed) (see Note 1). In order to distinguish between these two possibilities, he asked two questions about a ball that would be dropped from a window:

(1) Given the ball reaches the ground in $4 \mathrm{~s}$, how long would it take for this ball to get halfway to the ground?

(2) Given the window is 40 meters above the ground and it takes the ball $2 \mathrm{~s}$ to reach the ground, how far would the ball be from the ground after $1 \mathrm{~s}$ ?

If people's intuitive physics follows the Aristotelian model, the answer to the first and second questions should be $2 \mathrm{~s}$ and 20 meters, respectively. However, if it follows the Newtonian model, the answers should be "less than $2 \mathrm{~s}$ " and "less than 20 meters". The author observed about an equal number of Newtonian and Aristotelian answers. The author, however, admitted that Aristotelian responses might have been favored because they involve minimal computation. He thus conducted a further survey with multiple-choices that did not require such computations and observed fewer Aristotelian responses. In a final investigation, Shanon assessed people's perception of video-taped objects that were either free-falling or falling at a constant speed. Under this condition, his subjects correctly identified the free-falling objects as natural events. Thus the author noted a discrepancy between people's perception and cognition of falling objects because the latter appears not always to be consistent with the reality of the physical world.

A similar conclusion was reported by Vicovaro et al. (2019). Their participants rated the motion of a free-falling object as being more natural when it was accelerated than when the object moved with constant velocity. However, this effect was rather small (a difference of 5.3 points on a 100-point scale) and even absent for some combinations of object mass and motion velocity. In another study, Gravano et al. (2017) investigated mental imagery of throwing a ball against a ceiling under terrestrial gravity (with acceleration) and under microgravity (as during a space flight). Their results showed that participants' performance was mostly compatible with microgravity, both in the imagined terrestrial and the imagined space condition. These results suggest that under conditions of mental imagery, gravitational forces are not well represented. However, there is also evidence that these forces can be mentally represented. In one of the experiments of Huber and 
Krist (2004) participants were asked to estimate the flight time of a ball that rolled off a horizontal surface and fell-hidden from view-onto a landing point. The produced flight times showed a pattern that matched the normative rule, including the acceleration, very well. Thus, so far it is unclear whether the acceleration due to gravitational force is represented in the mental imagery of falling objects.

In the present study, we examined mental imagery of free fall in two different tasks, a height estimation, and a fall-time estimation task. Figure 1 shows two examples of the stimulus setup that was used for both tasks. In both tasks, participants were asked to imagine an apple falling from a certain height. In the height estimation task, participants were presented with different fall times and had to indicate the corresponding heights. In the fall-time estimation task, participants had to produce the fall time associated with free falls of different heights. The normative distance traveled by a falling object was calculated according to the law of free fall under two conditions, without and with air resistance. The law of free fall under conditions without air resistance states that $h(t)=\frac{1}{2} g t^{2}$, where $h$ denotes the height (i.e., free fall distance), $t$ the fall time, and $g$ the acceleration factor, which is $9.807 \mathrm{~m} / \mathrm{s}^{2}$. Rearranging this equation gives the fall time $t$ for a falling object to fall from height $h$, that is,

$$
t(h)=\sqrt{2 \frac{h}{g}} .
$$

Under the condition of air resistance, however, these functions become

$$
\begin{gathered}
h(t)=\frac{V_{\infty}^{2}}{g} \ln \cosh \frac{g \cdot t}{V_{\infty}} \\
t(h)=\frac{V_{\infty}}{g} \mathrm{a} \cosh \exp \frac{h \cdot g}{V_{\infty}^{2}}
\end{gathered}
$$

where $V_{\infty}$ denotes the terminal velocity that the object can reach, and which is given by

$$
V_{\infty}=\sqrt{\frac{2 \cdot r \cdot g}{c_{d} \cdot \rho \cdot A}} .
$$

In this equation, $m$ denotes the mass of the falling object, $A$ its cross-section area, $c_{d}$ the drag coefficient, and $\rho$ the density of air. The predicted functions for a falling apple without and with air resistance are shown in Fig. 2; note that $h(t)$ exhibits a convex shape, whereas $t(h)$ has a concave shape. 

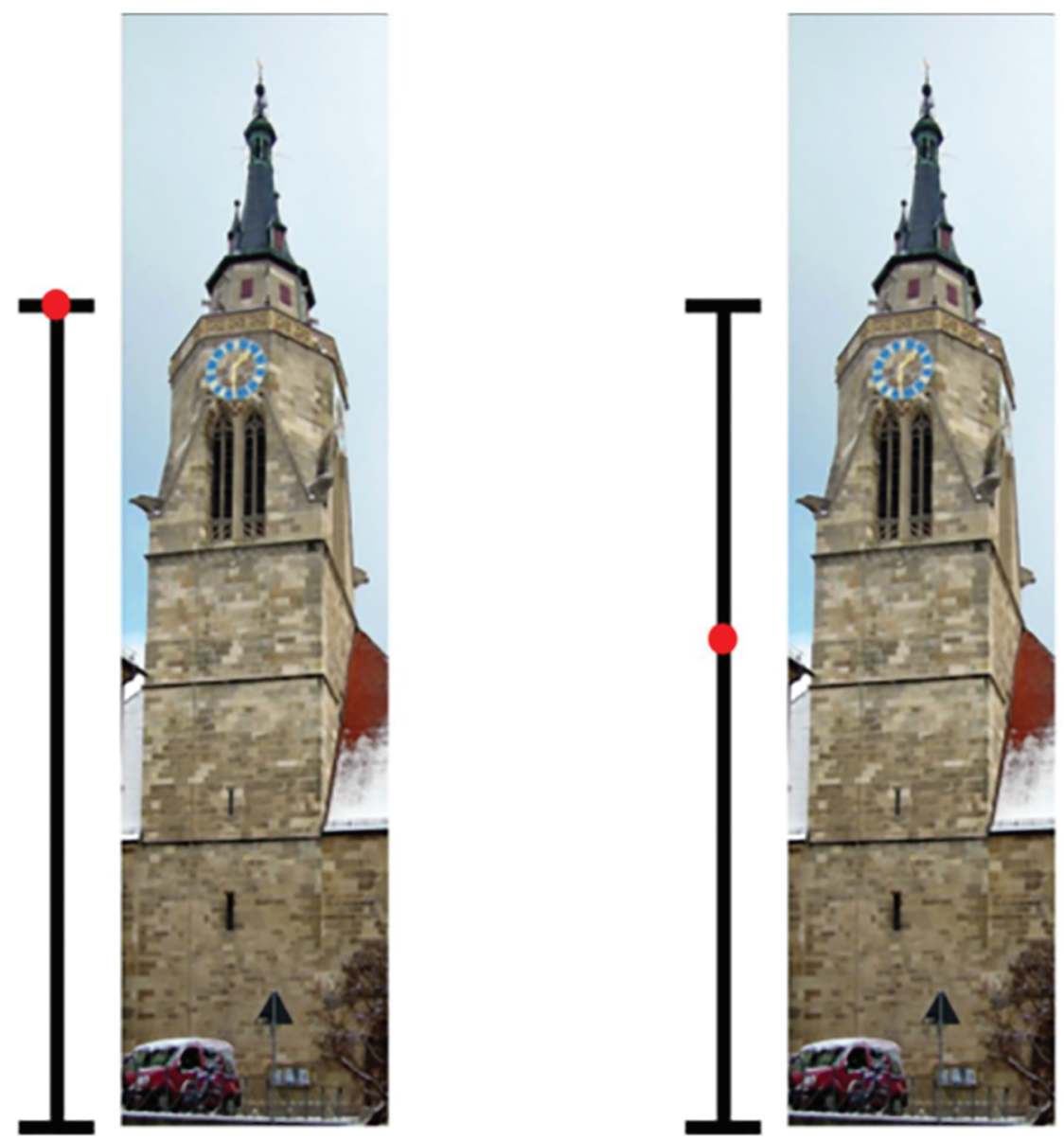

Figure 1. Two example height stimuli corresponding to $45 \mathrm{~m}$ (left) and $27 \mathrm{~m}$ (right) for both, the height estimation task and the fall-time estimation task. In both tasks, participants were asked to imagine an apple falling from the church's tower to the ground. In the height estimation task, participants heard a tone for the duration of a certain fall time, and then had to indicate the height from which the apple had fallen to the ground (by placing the red dot on the scale with a mouse click). In the fall-time estimation task, participants were presented with a certain height (indicated by the red dot) and then had to produce the imagined fall time (start and stop of a tone with a mouse click).

Accordingly, if participants' mental imagery of an object's (such as an apple's) free fall represents the acceleration due to gravitational force, we would expect a convex fall-time-height $h(t)$ function in the height estimation task, and a concave height-fall-time function $t(h)$ in the fall-time estimation task. Alternatively, participants' mental imagery might follow a linear relationship between height and fall time with constant velocity of object motion during free fall. 

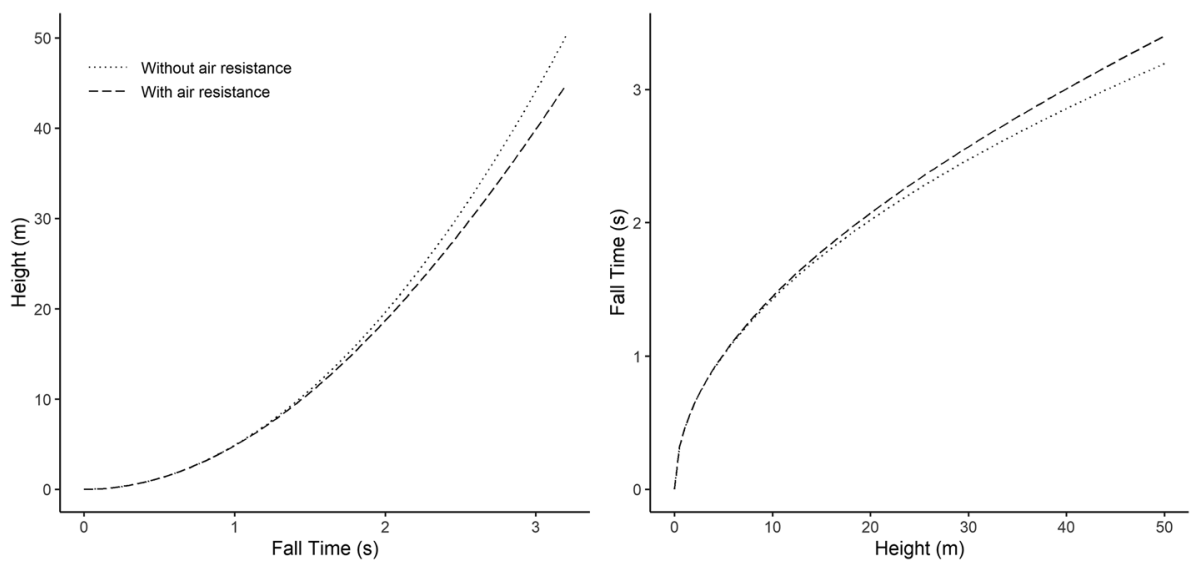

Figure 2. Illustration of functions $h(t)$ (left panel) and $t(h)$ (right panel) under the assumption of no air resistance and air resistance. We assume an apple with $m=0.15 \mathrm{~kg}$ and $d=7 \mathrm{~cm}$. The drag coefficient for a sphere is $c_{d}=0.5$, and the density of air is $\rho=1.2 \mathrm{~kg} / \mathrm{m}^{3}$.

\section{Material and Methods}

\subsection{Participants}

The final sample consisted of 40 participants (22 female) aged from 18 to 37 years $(M=21.52)$. Two additional participants were excluded because they apparently misunderstood the instruction of the height estimation task. Participants provided written informed consent and received course credit for their participation.

\subsection{Apparatus and Stimuli}

The experiment was programmed in PsychoPy v3.0.7 (Peirce et al., 2019). In both tasks, a reference height stimulus, a $16 \mathrm{~cm} \times 4.5 \mathrm{~cm}$ photograph of the tower of St. George's Collegiate Church (Stiftskirche) in Tübingen, was presented on a computer screen. This building was chosen because it is a very prominent building in Tübingen, which all residents and students should be familiar with. To the left of the photograph, a visual scale was presented, which ranged from the ground to the platform of the tower ( $45 \mathrm{~m}$ in reality), with markers on the lower and upper end. A red dot with a diameter of $0.5 \mathrm{~cm}$ was used to mark different heights on the scale, corresponding to 9, 18, 27, 36 or $45 \mathrm{~m}$ in reality. Auditory stimuli were presented via headphones (Sony MDR-XD200). A 300-Hz tone with an intensity of 67 $\mathrm{dB}(\mathrm{A})$ SPL served as stimulus for the normative fall times (without air resistance) corresponding to the five different heights $(1.355,1.916,2.346,2.709$, and $3.029 \mathrm{~s})$. Responses were collected with the left button of the computer mouse. 


\subsection{Procedure}

Each participant took part in one experimental session, which comprised a height estimation task and a fall-time estimation task. In both tasks, participants had to imagine the free fall of an apple from different heights. In the height estimation task, in each trial first a fixation cross was presented at the center of the screen for $1 \mathrm{~s}$. Then, the height reference stimulus (image of the church tower) and the visual scale were presented on the screen. When the participant clicked the mouse, a tone appeared with a duration of one of the five different fall times $(1.355,1.916$, $2.346,2.709$, or $3.029 \mathrm{~s}$ ). Then, the participant had to indicate with a mouse click on the scale the imagined height from which the apple had fallen. A red dot appeared on the indicated position (with no possibility of further adjustment), providing feedback that the height estimate was registered. In the fall-time estimation task, each trial started as in the height estimation task. That is, a fixation cross appeared for $1 \mathrm{~s}$ and then the height reference stimulus and the scale were presented. In contrast to the height estimation task, a red dot on the scale represented one of the five different heights $(9,18,27,36$ or $45 \mathrm{~m})$ and the participant had to imagine an apple falling from that height to the ground. The participant had to produce the imagined fall time with two mouse clicks (start and stop). During this interval, a continuous tone was played; however, the red dot on the scale representing the height of the apple remained static. When the participant did not stop the fall time within $10 \mathrm{~s}$, the trial was aborted and the next trial started.

The two tasks (height estimation vs. fall-time estimation) were administered in different halves of the experiment, and the order of the tasks was balanced across participants. Due to the exclusion of two participants, 21 participants started with the fall-time estimation task and 19 participants started with the height estimation task. For each of the two tasks, participants completed 75 experimental trials (15 trials for each height and fall time, respectively) in random order. At the beginning of each experimental half, participants completed five additional familiarization trials (randomly drawn from all possible trials).

\section{Results}

Two trials were excluded from the fall-time estimation data of one participant because they did not respond within the time out criterion (10 s) in these trials. In order to test for the shape of the estimated height-duration functions (accelerated vs. linear), we fitted second-order polynomials with the form $f(x)=a x^{2}+b x+c$ to the observed functions (separately for each participant and task). Then, we submitted the quadratic coefficients $a$ to a Bayesian 

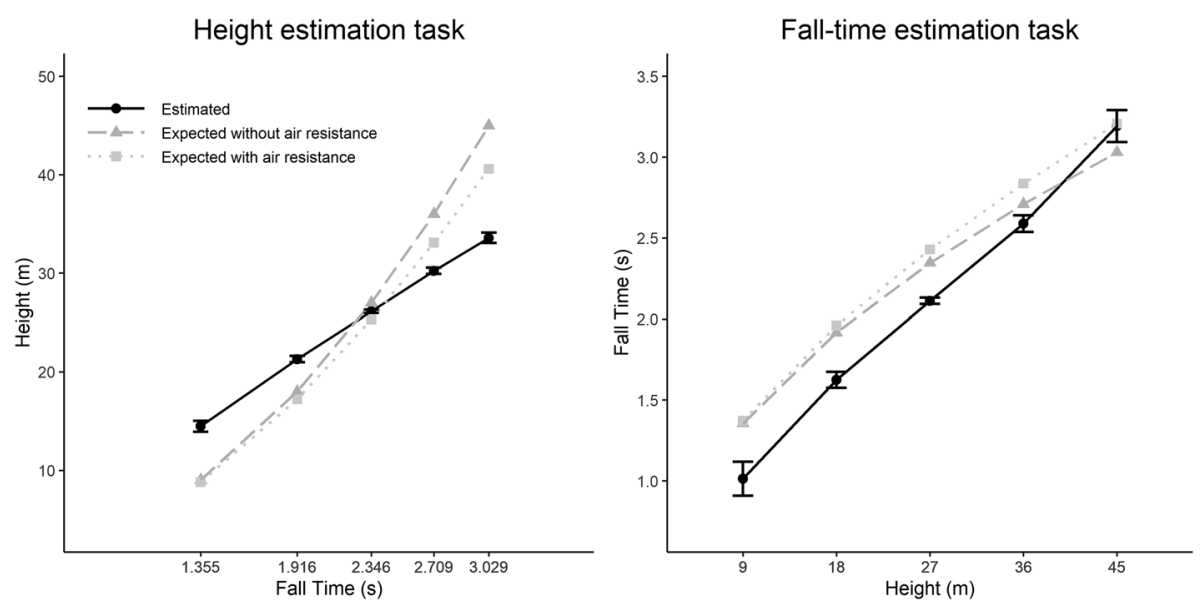

Figure 3. Left panel: Height estimates as a function of fall time in the height estimation task. Right panel: Fall-time estimates as a function of height in the fall-time estimation task. Error bars represent \pm 1 within-subject standard error, according to Morey (2008). Gray points show the expected heights and fall times according to the normative physical rule without air resistance (dotted) and with air resistance (dashed).

$t$-test (R package BayesFactor; Morey \& Rouder, 2018), testing the one-sided hypothesis that $a>0$ (convex shape) for height estimates and $a<0$ (concave shape) for fall-time estimates versus the hypothesis that $a=0$, respectively. The Bayes factor provides the ratio of the likelihood of the respective non-linear hypothesis $(a>0, a<0)$ to the likelihood of the linear hypothesis $(a=0)$ given the observed data (see e.g., Raftery, 1995). For example, a Bayes factor of 5 would mean that the linear hypothesis is five times more likely than the non-linear hypothesis, and a Bayes factor of 0.2 would mean that the linear hypothesis is five times less likely than the non-linear hypothesis.

Figure 3 shows estimated and normative functions in the height estimation task (left panel) and in the fall-time estimation task (right panel). The height estimates do not show the acceleration of the normative function. The estimated function, however, shows a slightly convex shape, that is, the estimated fall times represent a slightly decelerating rather than an accelerating function. Accordingly, the mean coefficient $a$ was negative $(M=-0.61, S E=0.39)$ and the Bayesian $t$-test revealed a Bayes factor of 0.07 (Note 2). In the fall-time estimation task, the deviation of the estimated from the expected function was less extreme than in the height estimation task. Nevertheless, there was also only very little evidence for an accelerated function in this task, with mean $a=-8.54 \times 10^{-6}\left(S E=9.72 \times 10^{-5}\right)$, and Bayes factor $=0.18$. 


\section{Discussion}

In the present study, we investigated whether people's mental imagery of free fall (of an object) represents the acceleration due to gravity. Irrespective of the estimation task (i.e., whether participants estimated heights or fall times), the results were more consistent with a linear than with an accelerated relationship between height and fall time. This suggests that the mental imagery of an object's free fall does not represent the acceleration due to gravity and thus resembles the Aristotelian rather than the Newtonian model of kinematic phenomena.

The present results are in line with the previous results by Gravano et al. (2017), who showed that the mental imagery of throwing a ball against the ceiling and catching it on the rebound was compatible with microgravity, irrespective of whether participants imagined the ball's motion under terrestrial or space conditions. They explained their results by assuming that participants used a visual mode of imagery, which does not represent the gravitational acceleration because visual processing of accelerating/decelerating motion is rather poor (e.g., Werkhoven et al., 1992). A related possibility why people do not represent the gravitational acceleration is that the Aristotelian model is an appropriate approximation of the largest parts of many free falls. Namely, under conditions of air resistance, falling objects rapidly attain a constant level of speed. Thus the initial phase of acceleration is too short to be carefully observed without instruments (see e.g., Rovelli, 2015). For example, the terminal velocity of an apple $(m=0.15 \mathrm{~kg}, d=7 \mathrm{~cm})$ would be $V_{\infty}=35.70 \mathrm{~m} / \mathrm{s}$. However, $75 \%$ of this terminal velocity is attained already after $3.54 \mathrm{~s}$ or $53.70 \mathrm{~m}$. According to this explanation, the mental representation of free fall does not include gravitational acceleration because the change of speed during the acceleration phase cannot be perceived. As a consequence, constant velocity is attributed to all phases of free fall.

As already mentioned in the Introduction, a study by Huber and Krist (2004) reported fall-time estimates that are consistent with an acceleration of imagined free fall. In their study, participants saw a ball rolling off a horizontal surface and had to estimate the time until the ball fell onto a marked landing point. Importantly, the fall of the ball was hidden from the participants' view by an occluding curtain so that they had to imagine the fall of the ball. In a production task, participants started the motion of the ball and had to indicate the point in time when the ball hit the ground. In a judgment task, participants had to judge the flight time by using a circular rating scale. In both tasks, the height and the distance to the landing point were manipulated. The produced flight times showed a pattern that matched the normative rule very well. In the judgment task, however, the judgments deviated from the predicted pattern. The authors explained this dissociation between produced and judged flight times by arguing that only in the production task (but not in the judgment task) mental imagery was based on a 
timing-responsive representation (e.g., Schwartz \& Black, 1999) of free fall, which helped to predict the point in time when the ball hit the ground.

One could argue that the production tasks used in Huber and Krist (2004) and the present study were comparable so that one should have expected accelerated functions also in the present study. There was, however, one possibly crucial difference between the two tasks. In the production task of Huber and Krist, the participants saw the ball moving on the horizontal surface until it was occluded, which was not the case for the apple in the current study (and also not in the judgment task of Huber and Krist). The perception of this initial movement of the ball might be crucial to activate dynamic mental imagery (see also Huber \& Krist, 2004). Thus, it remains unclear whether participants would represent the acceleration in the free-fall scenario of the present study if one provided a similar initial motion cue as in the study of Huber and Krist.

A related issue regarding the present study is whether participants actually imagined the free fall. Although they were instructed to do so, they could have followed the simple rule: the higher the height the longer the fall time and vice versa. Such a strategy would be virtually indistinguishable from mental imagery of a linear relationship between the two variables. Additionally, one could argue that the height estimation task used in the present study was not optimal for inducing mental imagery because participants had to indicate the height from which the apple fell. Possibly, mental imagery would have been easier or more natural in a scenario where the apple falls from a fixed height and participants indicate the height the apple reaches at the end of the free fall (even though this scenario can also be considered somewhat unnatural as the apple would not simply stop falling before it reaches the ground). In fact, two participants were excluded from data analyses because they apparently understood the task in the latter way. We used the former scenario because we wanted to make the height estimation task as similar to the fall-time estimation task as possible. In future studies, the assessment of eye movements could potentially shed some light on the involvement of mental imagery in free-fall scenarios like the present one (for a discussion of the relationship between eye movements and mental imagery, see Huber \& Krist, 2004).

In conclusion, the present study suggests that the intuitive physics of free fall as assessed by estimates of height and fall time during mental imagery of an object's free fall does not represent the gravitational acceleration. A plausible explanation for this result is that the change of speed during the rather short acceleration phase of free fall cannot be visually detected and hence people (incorrectly) attribute constant speed to all phases of free fall. Nevertheless, future studies may provide an answer to the question of whether people are capable of imaging the acceleration of falling objects when initial motion cues are available. 


\section{Acknowledgements}

We thank Stefan Köbke for building the experiment in PsychoPy and for assistance with data collection and two reviewers for their helpful comments.

\section{Notes}

1. For a review of intuitive physics about the shape of ballistic trajectories from Aristotle's times through the Middles Ages and malconceptions of simple ballistic motions, the interested reader should consult Hecht and Bertamini (2000).

2. Since the numerical mean value of $a$ was negative, we also conducted a Bayesian $t$-test, testing the hypothesis that $a<0$ against the hypothesis that $a=0$. This analysis yielded a Bayes factor of 1.03 .

\section{References}

Gravano, S., Zago, M., \& Lacquaniti, F. (2017). Mental imagery of gravitational motion. Cortex, 95, 172-191. doi: 10.1016/j.cortex.2017.08.005.

Hecht, H., \& Bertamini, M. (2000). Understanding projectile acceleration. J. Exp. Psychol. Hum. Percept. Perform., 26, 730-746. doi: 10.1037/0096-1523.26.2.730.

Huber, S., \& Krist, H. (2004). When is the ball going to hit the ground? Duration estimates, eye movements, and mental imagery of object motion.J. Exp. Psychol. Hum. Percept. Perform., 30, 431-444. doi: 10.1037/0096-1523.30.3.431.

Kubricht, J. R., Holyoak, K. J., \& Lu, H. (2017). Intuitive physics: Current research and controversies. Trends Cogn. Sci., 21, 749-759. doi: 10.1016/j.tics.2017.06.002.

McCloskey, M., Washburn, A., \& Felch, L. (1983). Intuitive physics: The straight-down belief and its origin. J. Exp. Psychol. Learn. Mem. Cogn., 9, 636-649. doi: 10.1037//0278-7393.9.4.636.

Morey, R. D. (2008). Confidence intervals from normalized data: A correction to Cousineau (2005). Tutor. Quant. Methods Psychol., 4, 61-64.

Morey, R. D., \& Rouder, J. N. (2018). BayesFactor: Computation of Bayes Factors for Common Designs. R package version 0.9.12-4.2. Retrieved from https://CRAN.R-project.org/package=BayesFactor.

Oberle, C. D., McBeath, M. K., Madigan, S. C., \& Sugar, T. G. (2005). The Galileo bias: A naive conceptual belief that influences people's perceptions and performance in a ball-dropping task. J. Exp. Psychol. Learn. Mem. Cogn., 31, 643-653. doi: 10.1037/0278-7393.31.4.643.

Peirce, J., Gray, J. R., Simpson, S., MacAskill, M., Höchenberger, R., Sogo, H., Kastman, E., \& Lindeløv, J. K. (2019). PsychoPy2: Experiments in behavior made easy. Behav. Res. Methods, 51, 195-203. doi: 10.3758/s13428-018-01193-y.

Raftery, A. E. (1995). Bayesian model selection in social research. Sociol. Methodol., 25, 111-163. doi: $10.2307 / 271063$.

Rohrer, D. (2002). Misconceptions about incline speed for nonlinear slopes. J. Exp. Psychol. Hum. Percept. Perform., 28, 963-973. doi: 10.1037/0096-1523.28.4.963. 
Rovelli, C. (2015). Aristotle's physics: a physicist's look.J. Am. Philos. Assoc., 1, 23-40. doi: 10.1017/ apa.2014.11.

Schwartz, D. L., \& Black, T. (1999). Inferences through imagined actions: Knowing by simulated doing. J. Exp. Psychol. Learn. Mem. Cogn., 25, 116-136. doi: 10.1037/0278-7393.25.1.116.

Shanon, B. (1976). Aristoteliansim, Newtonianism and the physics of the layman. Perception, 5, 241-243. doi: 10.1068/p050241.

Vicovaro, M., Noventa, S., \& Battaglini, L. (2019). Intuitive physics of gravitational motion as shown by perceptual judgment and prediction-motion tasks. Acta Psychol., 194, 51-62. doi: 10.1016/j. actpsy.2019.02.001.

Werkhoven, P., Snippe, H. P., \& Toet, A. (1992). Visual processing of optic acceleration. Vision Res., 32, 2313-2329. doi: 10.1016/0042-6989(92)90095-Z. 